\title{
The Importance of Cycle Threshold Values in the Evaluation of Patients with Persistent Positive PCR for SARS-CoV-2: Case Study and Brief Review
}

\author{
Jose Bordon ${ }^{1,2 *}$, MD PhD; Donghoon Chung ${ }^{3}$, PhD; Priya Krishnan ${ }^{4}, M D ;$ Ruth Carrico ${ }^{1}, P h D ;$ Julio A. Ramirez ${ }^{1}, M D$ \\ ${ }^{1}$ Division of Infectious Diseases, Department of Medicine, University of Louisville School of Medicine, Louisville, KY, USA; ${ }^{2}$ Washington Health Institute, Washington, \\ D.C., USA; ${ }^{3}$ Department of Microbiology and Immunology, University of Louisville School of Medicine, Louisville, KY, USA; ${ }^{4}$ Division of General Internal Medicine,
} Department of Medicine, University of Louisville School of Medicine, Louisville, KY, USA

*jbordon@dc-whi.org

Recommended Citation: Bordon J, Chung D, Krishnan P, Carrico R, Ramirez JA. The importance of cycle threshold values in the evaluation of patients with persistent positive PCR for SARS-CoV-2: Case study and brief review. Univ Louisville J Respir Infect 2020; 4(1):Article 54. doi: 10.18297/jri/vol4/iss1/54.

\begin{abstract}
Some patients recover from COVID-19, but their reverse transcription-polymerase chain reaction (RT-PCR) for SARSCoV-2 remains persistently positive. In the evaluation of these patients, it is important to define the cycle threshold (Ct) value of the RT-PCR test. This article will present a case study, ad-
\end{abstract}

dress relevant findings and interpretation of the RT-PCR test, and explain the use of $\mathrm{Ct}$ values in defining when a healthcare worker may return to work. Our current approach is to allow health care workers with persistently positive RT-PCR to return to work if the $\mathrm{Ct}$ value is greater than 35 .

\section{Introduction}

The coronavirus disease of 2019 (COVID-19) pandemic is causing a shocking death toll in large because of the high transmissibility of the severe acute respiratory syndrome coronavirus 2 (SARS-CoV-2) and the severity of illness in about $20 \%$ of cases.[1] It is imperative to have a reliable test for early identification of individuals with SARS-CoV-2 infection. A reliable SARS-CoV2 test will be instrumental for identification of people with the infection, preventing the spread and mitigating the epidemic.[2] Additionally, a highly reliable test will allow early interventions that include isolation and appropriate medical care of the infected persons that in turn will be translated into the protection of the community at risk.

In a short period of time, there has been substantial progress in the confirmation of COVID-19 by a positive SARS-CoV-2 reverse transcription polymerase chain reaction (RT-PCR) test in a symptomatic patient.[3] However, there are important uncertainties in relation to the best interpretation of the test in different scenarios.[4] It remains to be determined what is the best interpretation of a negative RT PCR assay in a symptomatic patient. It is becoming an established practice to use two consecutive negative respiratory samples for SARS-CoV-2 at intervals of 24 hours or longer for discontinuation of isolation in a convalescent patient with COVID-19.[5] Unfortunately, there are some challenges in the best interpretation of test results including communication with high risk individuals (e.g. elderly with major comorbidities, etc), those hospitalized patients stable enough to return to or enter chronic care facilities, and those recovering from the COVID-19 with persistent positive tests. This article will present a case study and address relevant findings and interpretation of the diagnostic RT-PCR test in respiratory specimens to assist clinicians in their communications and action plans.

\section{Case Study}

A 39-year old registered nurse was evaluated at the hospital employee health clinic due to sore throat and fever. She works on the general medicine ward. During her evaluation, a nasopharyngeal swab sample was obtained for SARS CoV-2 RT-PCR. The result of the RTPCR test was positive. She was not complaining of cough or shortness of breath. Her social history and past medical history were unremarkable. Her temperature was $101.5^{\circ} \mathrm{F}$, heart rate $95 \mathrm{bpm}$, repiratory rate 18 bpm, blood pressure $125 / 80 \mathrm{mmHg}$, and oxygen sat- 


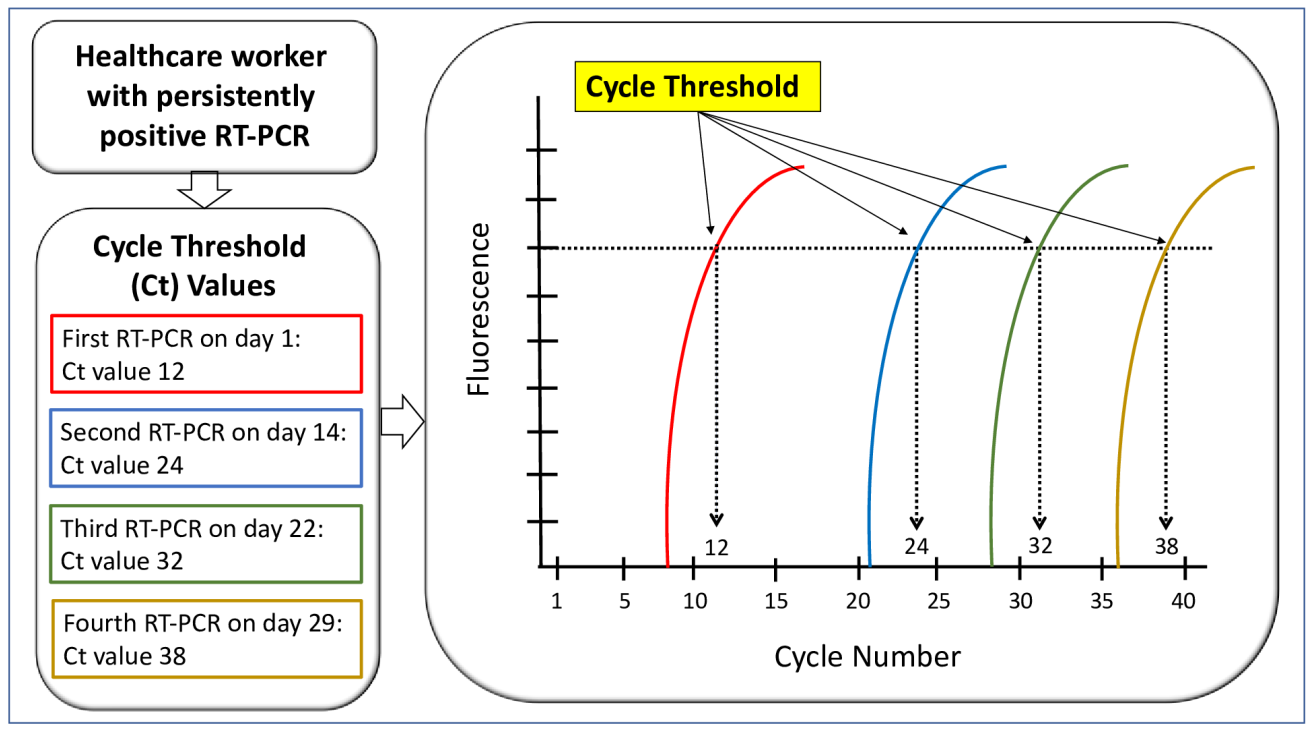

Figure 1. Schematic representation of the cycle threshold values for the health care worker described in the case study.

uration of $96 \%$ on room air. The final diagnosis was COVID-19. She was advised to begin home isolation, to call if any clinical deterioration, and to plan on returning to clinic in 14 days for evaluation before returning to work. When she returned to the clinic on day 14 , she was afebrile and had been asymptomatic for 8 days. The repeat SARS CoV-2 RT-PCR was positive. The patient returned to clinic on day 22 , and the SARS CoV-2 RT-PCR was again positive. She was advised to return to clinic again in 7 days. On day 29 , the repeat RT-PCR was still positive and at this time the patient had been asymptomatic for 23 days. The hospital policy stated that a healthcare worker needed to have a demonstrated negative RT-PCR before returning to work. To further evaluate the case, the cycle threshold $(\mathrm{Ct})$ values were collected for each of the four RT-PCR tests. A graphic representation of the $\mathrm{Ct}$ values for the patient are depicted in Figure 1.

Since the last Ct value was elevated at 38, it was considered that the patient was no longer infectious and she was allowed to return to work.

\section{SARS-CoV-2 RT-PCR Assay}

There are many internal and external factors of the SARS-CoV-2 RT PCR test accounting for the optimal diagnostic yield.

\section{Internal factors}

This paper is directed to clinicians therefore some internal factors of the SARS-CoV-2 RT PCR assay are beyond the aim of this paper. The main internal factors of the
RT PCR include the target genes and cycle threshold $(\mathrm{Ct})$.

Genes target for the SARS-CoV-2 assays: Currently the most sensitive SARS-CoV-2 RT PCR assays use the genes N1, N2 and E. The Centers for Disease Control and Prevention (CDC) recommends the genes N1 and $\mathrm{N} 2$ encoding the two nucleocapsid proteins and the human RNase $\mathrm{P}$ gene to confirm the ribonucleic acid (RNA) extraction. On the other hand, the World Health Organization (WHO) recommends the assay using the gene E; which requires a confirmatory assay using the RdRp gene. Both assays have high analytic sensitivity and specificity for SARS-CoV-2.[6] These two assays are highly specific for SARS-CoV-2 with no cross-reactivity with other respiratory viruses as reported by Nalla et al. in an evaluation of assays using seven different primerprobe sets and one assay kit.[7]

The basics on the cycle threshold: Real-time assay is a type of PCR assay where positive reactions (nucleic acid amplifications) are detected by accumulation of the fluorescent signal in real-time. As the cycles of the real-time PCR continue, the fluorescent signal increases exponentially by two-fold and it becomes significantly higher from the background, called 'threshold'. The cycle number at which the signal crosses the threshold is called a cycle threshold (Ct) (Figure 1). Ct levels are inversely proportional to the amount of the target nucleic acid in the sample. This means that the lower the $\mathrm{Ct}$ value the greater the amount of the target nucleic acid in the sample and vice-versa.

The cut off for positive and negative $\mathrm{Ct}$ for SARS CoV-2 remains unclear. However, a good number of authors 
recommend a cut off of $40 .[2,6,8]$ It means a test is considered positive if the $\mathrm{Ct}$ is $<40$ and vice-versa. On the other hand, the European Centre for Disease Prevention and Control (ECDC) considers the Ct cut off of 35 and a $\mathrm{Ct}$ value $>35$ considers that it could be the result from a contaminant. ECDC recommends all positive results to be confirmed by a second gene target.[9] Holshue et al. reported the first case of COVID-19 in the US and the NP Cts on the days 4, 7, 11 and 12 were 12-18, 23-24, 33-34 and 37-40 respectively.[10] Similar trend of increasing $\mathrm{Ct}$ values over time were reported by others.[11]

\section{External factors}

Biological specimens: Samples from the respiratory tract are the most recommended specimens to test for SARS-CoV-2. A nasopharyngeal specimen (NP) is the preferred choice for swab-based SARS-CoV-2 testing, but oropharyngeal, mid-turbinate, anterior nares samples, sputum and tracheal aspirate and bronchoalveolar lavage also are acceptable specimens. However, the yield of a positive test is not the same for each type of specimen and also it depends on the time of the course of the infection.[12] The yield for positive tests were reported to be greater for sputum and bronchoalveolar lavage (BAL) than the NP, OP and nasal swabs.[12] Improper collection of respiratory specimens could be sources of false-negative tests.

\section{SARS-CoV-2 RT-PCR and Timing of Testing}

Positive SARS-CoV-2 RT-PCR results have been reported during the incubation time of COVID-19 when patients are asymptomatic, during the symptomatic period and lastly during convalescence time when the patients become asymptomatic. On the other hand, there are pending answers to the interpretation of the results of SARS-CoV-2 RT-PCR during these three time points of the illness and its translation to infectivity.

\section{RT-PCR test during the asymptomatic infection}

Rothe et al. reported SARS-CoV-2 RT-PCR from an asymptomatic individual who turned to be the original source of infection, also known as "the index case", for an individual who became the source for infection of 3 other individuals. The transmissions from the asymptomatic index case to patient 1 and from patient 1 to patients 2 to 4 occurred in a total of 7 days. All these persons including the index case subsequently developed mild illness characterized by fever and mild respiratory symptoms and all had positive SARS-CoV-2 from respiratory sources. In this series, one person had a viral load of 108 copies/ml of sputum.[13] The outbreak of COVID-19 on the cruise ship Diamond Princess illustrates the SARS-CoV-2 among asymptomatic persons. This outbreak resulted in the SARS-CoV-2 infec- tion of $712(19.18 \%)$ persons out of 3,711 passengers and crew members.[14] Ninety (2.4\%) persons had asymptomatic SARS-CoV-2 infection with a median age of 59.5 years. Among the asymptomatic persons, $20 \%$ had arterial hypertension and $9 \%$ diabetes mellitus. The median number of days between the first positive PCR test and the first of the two serial negative PCR tests was 9 days. These results of positive SARS-CoV-2 RTPCR highlights the unique nature of COVID-19, which can be asymptomatic and contagious. The confirmation of asymptomatic COVID-19 transmitting the infection to others demands to consider universal testing as a strategy to identify the source of infection.

\section{RT-PCR test during the symptomatic infection}

Zou et al. monitored SARS-CoV-2 viral load of nasal and swab swabs from 18 patients in relation to the symptom onset.[15] Lower Ct, higher viral load, were detected soon after the symptoms onset. Interestingly, the viral loads and $\mathrm{Ct}$ were similar regardless of the presence of the symptoms. In most individuals with symptomatic COVID-19 infection, the viral RNA is detectable as early as day 1 of symptoms and peaks within the first week of symptom onset in nasopharyngeal swab.[11] In patients without severe COVID-19, the SARS-CoV-2 detection declines by week 3 to undetectable. On the other hand, in severe COVID-19 the SARS-CoV-2 detection may persist longer than 3 weeks after illness onset. It remains to be determined whether the persistent SARS-CoV-2 is viral infectivity.[11, 16]

RT-PCR test after the symptomatic infection or convalescence

Interpreting the result of a SARS-CoV-2 test depends on the accuracy of the test and the pre-test probability or estimated risk of disease before testing. Pre-test probability is in relation to the prevalence of the illness under evaluation and it influences the accuracy of the test result. The SARS-CoV-2 RT-PCR tests were reported to have a sensitivity of $70 \%$ and a specificity of $98 \%$ [6 ,16]. SARS-CoV-2 RT-PCR has been reported to remain positive in sputum and BAL when the NP swabs become negative.[11] Positive SARS-CoV-2 viral cultures have not been reported after day 8 of symptoms onset.[11, 16] Though positive SARS-CoV-2 RT-PCR has been reported beyond many weeks following the first positive test, it is tempting to think that positive SARSCoV-2 RT-PCR results after day 8 of symptom onset may not be infectious. Persistent positive tests have been reported in some conditions that were not infections e.g. persistent positive acid-fast bacilli on staining test paired to negative cultures in patients receiving treatment for tuberculosis with resolving symptoms. 


\section{SARS-CoV-2 RT-PCR Sensitivity and Specificity}

Because PCR generates two copies of product at each cycle, the difference of 1 in $\mathrm{Ct}$ value indicates a difference of the target by a two-fold in the input sample. For example, a sample with a Ct of 20 might have 1024-fold higher amount of copy number than that with a $\mathrm{Ct}$ of 30 (230-20). Low Ct values clearly indicate the presence of high target copy numbers in the sample; however, high $\mathrm{Ct}$ values (weak positives) could be due to artifactual effects, such as noisy signal, random amplification, or potential contamination. All positive results should therefore be confirmed by a second gene target. For this reason, most of real-time PCR test for SARS-CoV-2 use two targets. Only samples with positive results for the both of two targets are determined as positive for SARS-CoV-2. In case only one of the two is positive, it is considered as "undetermined" and recommended for a second test.

Generally, a real-time PCR shows an extremely high sensitivity, with a detection limit of 1-10 copies per sample and this holds true for the published real-time PCR assays for SARS-CoV2. This means that a sample with a Ct at the cut-off for positive (e.g., 40) might have 1-10 copies of RNA in the sample. Considering the fact that the target of the most of real-time PCR assays for SARS-CoV2 is $\mathrm{N}$ gene, which is present in all species of viral mRNA and genomic RNA, high Ct values should be carefully interpreted as it cannot indicate the presence of infectious virus or active replications (i.e., genomic RNA). In an unpublished study for our group, noinfectious viruses were rescued from clinical NP samples that have $\mathrm{Ct}>30$.

Positive SARS-CoV-2 RT-PCR and infectivity: Bullard et al. examined the relationship between SARSCoV-2 RT-PCR Ct values from respiratory samples, symptom onset to test (STT) and infectivity in Vero cell lines culture.[16] Viral growth was seen in only $28.9 \%$ of samples. Interestingly, there was no growth in samples with a $\mathrm{Ct}>24$ or STT $>8$ days. They concluded that infectivity may be low in patients with $\mathrm{Ct}>24$ and duration of symptoms longer than 8 days. Similarly, Wolfel et al. reported no SARS-CoV-2 grows in specimens collected after 8 days of the onset of symptoms.[11]

\section{Conclusion}

$\mathrm{Ct}$ values appear to play an important role when the test-based discontinuation of isolation of the patient is considered. It appears that infectivity is low in patients with $\mathrm{Ct}$ values greater than 24 . In the case study presented in this manuscript, the healthcare worker was asymptomatic with a $\mathrm{Ct}$ value of 38 at day 29 after the initial positive RT-PCR. It was considered that the positive RT-PCR likely reflected the presence of residual viral genomic RNA, without the presence of live viruses. The healthcare worker was deemed non-infectious and she was allowed to return to work. Our current approach is to allow to return to work healthcare workers with persistently positive RT-PCR if the Ct values are greater than 35 .
Received: July 17, 2020

Accepted: July 172020

Published: July 24, 2020

Copyright: (C) 2022 The author(s). This original article is brought to you for free and open access by ThinkIR: The University of Louisville's Institutional Repository. For more information, please contact thinkir@louisville.edu. This article is distributed under the terms of the Creative Commons Attribution 4.0 International License (CC BY 4.0), which permits unrestricted use, distribution, and reproduction in any medium, provided the original author and source are credited.

Funding Source: The author(s) received no specific funding for this work.

Conflict of Interest: All authors declared no conflict of interest in relation to the main objective of this work.

\section{References}

1. Centers for Disease Control and Prevention (CDC). Coronavirus disease 2019 (COVID-19): Cases in the U.S. Available at: https://www.cdc.gov/coronavirus/2019-ncov/casesupdates/cases-in-us.html. Accessed 15 July 2020.

2. Tang YW, Schmitz JE, Persing DH, Stratton CW. Laboratory diagnosis of COVID-19: Current issues and challenges. J Clin Microbiol 2020; 58(6). doi: 10.1128/jcm.00512-20. PMID: 32245835.

3. Lu X, Wang L, Sakthivel SK, et al. US CDC real- time reverse transcription PCR panel for detection of severe acute respiratory syndrome coronavirus 2. Emerg Infect Dis 2020; 26(8):1654-65. doi: 10.3201/eid2608.201246. PMID: 32396505 .

4. Department of Health and Human Services USA. Guidance on interpreting COVID-19 test results. Available at: https://www.whitehouse.gov/wp-content/uploads/ 2020/05/Testing-Guidance.pdf. Accessed 15 July 2020.

5. Centers for Disease Control and Prevention (CDC). Cri- 
teria for return to work for healthcare personnel with suspected or confirmed COVID-19 (interim guidance). Available at: https://www.cdc.gov/coronavirus/2019-ncov/hcp/returnto-work.html. Accessed 15 July 2020.

6. Cheng MP, Papenburg J, Desjardins M, et al. Diagnostic testing for severe acute respiratory syndrome-related coronavirus 2: A narrative review. Ann Intern Med 2020; 172(11):726-34. doi: 10.7326/m20-1301. PMID: 32282894.

7. Nalla AK, Casto AM, Huang MW, et al. Comparative performance of SARS-CoV-2 detection assays using seven different primer-probe sets and one assay kit. J Clin Microbiol 2020; 58(6). doi: 10.1128/jcm.00557-20. PMID: 32269100.

8. Woloshin S, Patel N, Kesselheim AS. False negative tests for SARS-CoV-2 infection - challenges and implications. N Engl J Med 2020; 383(6):e38. doi: 10.1056/NEJMp2015897. PMID: 32502334.

9. European Center for Disease Prevention and Control. Questions and answers regarding laboratory topics on SARS-CoV-2. Available at: https://www.ecdc.europa. eu/en/all-topics-z/coronavirus/threats-and-outbreaks/covid19/laboratory-support/questions. Accessed 15 July 2020.

10. Holshue ML, DeBolt $C$, Lindquist $S$, et al. First case of 2019 novel coronavirus in the United States. N Engl J Med 2020; 382(10):929-36. doi: 10.1056/NEJMoa2001191. PMID: 32004427.
11. Wölfel R, Corman VM, Guggemos W, et al. Virological assessment of hospitalized patients with COVID-2019. Nature 2020; 581(7809):465-9. doi: 10.1038/s41586-020-2196x. PMID: 32235945.

12. Wang $W, X u Y$, Gao $R$, et al. Detection of SARSCoV-2 in different types of clinical specimens. JAMA 2020; 323(18):1843-4. doi: 10.1001/jama.2020.3786. PMID: 32159775.

13. Rothe $C$, Schunk $M$, Sothmann $P$, et al. Transmission of 2019-nCoV infection from an asymptomatic contact in Germany. N Engl J Med 2020; 382(10):970-1. doi: 10.1056/NEJMc2001468. PMID: 32003551.

14. Sakurai A, Sasaki $T$, Kato $S$, et al. Natural history of asymptomatic SARS-CoV-2 infection. N Engl J Med 2020; 383(9):885-6. doi: 10.1056/NEJMc2013020. PMID: 32530584.

15. Zou L, Ruan F, Huang M, et al. SARS-CoV-2 viral load in upper respiratory specimens of infected patients. $\mathrm{N}$ Engl J Med 2020; 382(12):1177-9. doi: 10.1056/NEJMc2001737. PMID: 32074444.

16. Bullard J, Dust K, Funk D, et al. Predicting infectious severe acute respiratory syndrome coronavirus 2 from diagnostic samples. Clin Infect Dis 2020; 71(10):2663-6. doi: 10.1093/cid/ciaa638. PMID: 32442256. 